\title{
Measuring 4th Generation CKM Parameters at the LHC
}

\section{George Wei-Shu Hou*i}

Department of Physics, National Taiwan University, Taipei, Taiwan 10617

National Center for Theoretical Sciences, National Taiwan University, Taipei, Taiwan 10617

E-mail: wshoudphys.ntu.edu.tw

With search for 4th generation $t^{\prime}$ and $b^{\prime}$ quarks continuing at the LHC, we note that the measurements of CPV phase in $B_{s} \rightarrow J / \psi \phi$ and the $B_{s} \rightarrow \mu^{+} \mu^{-}$rate would provide a measurement of $V_{t^{\prime} s}^{*} V_{t^{\prime} b}$. The third measurement of $A_{\mathrm{FB}}\left(B^{0} \rightarrow K^{* 0} \mu^{+} \mu^{-}\right)$would provide further information. Summer 2011 data reveal that $t^{\prime}$ and $b^{\prime}$ quark mass bounds are already rather close to the unitarity bound, while all B physics results (except $A_{S L}$ of D0) are now consistent with the 3 generation Standard Model. The implications of these results are presented, in particular giving an illustrative $b \rightarrow s$ quadrangle that may be relevant for the Baryon Asymmetry of the Universe. With $\left|V_{t^{\prime} s}^{*} V_{t^{\prime} b}\right|$ likely smaller than indicated by past data, we point out that a measurement of $\mathscr{B}\left(t^{\prime} \rightarrow b^{\prime} W^{*}\right.$ ) (or $\left.\mathscr{B}\left(b^{\prime} \rightarrow t^{\prime} W^{*}\right)\right)$ could be an excellent probe of rather small $V_{t^{\prime} b}\left(\right.$ or $\left.V_{t b^{\prime}}\right)$.

The 2011 Europhysics Conference on High Energy Physics-HEP 2011,

July 21-27, 2011

Grenoble, Rhône-Alpes France

\footnotetext{
* Speaker.

${ }^{\dagger}$ I thank my collaborators Masaya Kohda and Fanrong Xu on arXiv:1107.2343 [hep-ph], and Yuan Chao, Kai-Feng Chen, Shing-Kuo Chen, Yeong-Jyi Lei, and especially Bo-Yan Huang, on Phys. Rev. D 84, 014029 (2011).
} 


\section{Introduction}

CMS has put forth stringent mass bounds of $m_{t^{\prime}}>450 \mathrm{GeV}$ [四] and $m_{b^{\prime}}>495 \mathrm{GeV}$ at $90 \%$ C.L. []. We show that, concurrent with direct $t^{\prime}$ and $b^{\prime}$ search, measurement of CPV phase $\sin 2 \Phi_{B_{s}} \equiv \sin \phi_{s}$ in $B_{s} \rightarrow J / \psi \phi$, as well as the $B_{s} \rightarrow \mu^{+} \mu^{-}$rate, could give us $V_{t^{\prime} s}^{*} V_{t^{\prime} b}$ that could touch [B] upon the baryon asymmetry of the Universe (BAU).

\section{Measuring $V_{t^{\prime} s}^{*} V_{t^{\prime} b}$ via $\sin 2 \Phi_{B_{s}}$ and $\mathscr{B}\left(B_{s} \rightarrow \mu^{+} \mu^{-}\right)$}

The $B_{s}$ mixing amplitude $M_{12}^{s}=\frac{G_{F}^{2} M_{W}^{2}}{12 \pi^{2}} m_{B_{s}} f_{B_{s}}^{2} \hat{B}_{B_{s}} \eta_{B} \Delta_{12}^{s}$ is modified by the presence of $t^{\prime}$, i.e.

$$
\Delta_{12}^{s}=\left(\lambda_{t}^{\mathrm{SM}}\right)^{2} S_{0}(t, t)+2 \lambda_{t}^{\mathrm{SM}} \lambda_{t^{\prime}} \Delta S_{0}^{(1)}+\lambda_{t^{\prime}}^{2} \Delta S_{0}^{(2)},
$$

where $\lambda_{q} \equiv V_{q s}^{*} V_{q b}$, and $\Delta S_{0}^{(i)}$ [四] are GIM-subtracted $t^{\prime}$ effects. Unlike $\Delta m_{B_{s}} \equiv 2\left|M_{12}^{s}\right|$, the CPV phase $2 \Phi_{B_{s}}=\arg \Delta_{12}^{s}$ is purely short distance. Normalizing [回] the $B_{s} \rightarrow \mu^{+} \mu^{-}$rate by $\Delta m_{B_{s}}$,

$$
\mathscr{B}\left(B_{s} \rightarrow \bar{\mu} \mu\right)=\frac{3 g_{W}^{4} m_{\mu}^{2}}{2^{7} \pi^{3} M_{W}^{2}} \frac{\tau_{B_{s}} \eta_{Y}^{2}}{\hat{B}_{B_{s}} \eta_{B}} \frac{\left|\lambda_{t}^{\mathrm{SM}} Y_{0}\left(x_{t}\right)+\lambda_{t^{\prime}} \Delta Y_{0}\right|^{2}}{\left|\Delta_{12}^{s}\right| /\left.\Delta m_{B_{s}}\right|^{\exp }},
$$

where $\Delta Y_{0}$ is similar to $\Delta S_{0}$, the $f_{B_{s}}$ dependence is removed, making it another useful measurable.

The summer 2011 result for $\sin \phi_{s}$ by LHCb did not support the indication, since 2008, of a large and negative value. But -0.3 is still allowed at $\sim 2 \sigma$. Thus, we take two possible values

$$
\sin 2 \Phi_{B_{s}}=-0.3 \pm 0.1 ;-0.04 \pm 0.1 \quad\left(\mathrm{LHCb}>1 \mathrm{fb}^{-1}\right) .
$$

The combined $B_{s} \rightarrow \mu^{+} \mu^{-}$rate of LHCb and CMS is now only 3.4 times SM value, hence we take

$$
10^{9} \mathscr{B}\left(B_{s} \rightarrow \mu^{+} \mu^{-}\right)=5.0 \pm 1.5 ; 2.0 \pm 1.5 \quad \text { (2012), }
$$

which are adjacent regions that intersect at the SM rate. The numbers and errors in Eqs. (2.3]) and (R.4) anticipate possible developments in 2012. Together we have 4 cases: Case A when both high; Case B (C) for Eq. ([.3) SM-like, but Eq. (2.4) higher (lower) than SM; and Case D. In Fig. W we illustrate Case A in the $\phi_{s b} \equiv \arg V_{t^{\prime} s}^{*} V_{t^{\prime} b}, r_{s b} \equiv\left|V_{t^{\prime} s}^{*} V_{t^{\prime} b}\right|$ plane for $m_{t^{\prime}}=550 \mathrm{GeV}$, with the overlap of contours marked as A, and likewise for Cases B and C. We see that these cases cover a good part of the region for $\left|V_{t^{\prime} s}^{*} V_{t^{\prime} b}\right| \lesssim 0.01$, with Case $\mathrm{D}$ in fact a sliver that is complement to Case A.

\section{Utility of $A_{\mathrm{FB}}\left(B^{0} \rightarrow K^{* 0} \mu^{+} \mu^{-}\right)$; and Measuring $\left|V_{t^{\prime} b}\right|$ by Threshold Effect}

Contrary to earlier indications, $\mathrm{LHCb}$ again found $A_{\mathrm{FB}}\left(B^{0} \rightarrow K^{* 0} \mu^{+} \mu^{-}\right)$consistent with SM. In left plot of Fig. 2, Cases A-C are compared with contours of zero crossing point $\left.s_{0} \equiv q^{2}\right|_{A_{\mathrm{FB}}=0}$ (insensitive to form factors), where $s_{0}^{\mathrm{SM}} \simeq 4.4 \mathrm{GeV}^{2}$. For the four sample points, marked as small ellipses from high to low $r_{s b}$, the differential $A_{\mathrm{FB}}$ curves are given in center plot of Fig. 2 from top to bottom. The black solid curve is for SM. We see that the upper two curves or sample points are ruled out by LHCb data, implying that $\left|V_{t^{\prime} s}^{*} V_{t^{\prime} b}\right| \lesssim 0.008$ for $m_{t^{\prime}}=550 \mathrm{GeV}$. Note that for higher $m_{t^{\prime}}$ values, the formulas we use may no longer apply, as $t^{\prime}, b^{\prime}$ Yukawa couplings turn nonperturbative.

Since $V_{t^{\prime} b}$ could now be rather small, we mention that a measurement of $\mathscr{B}\left(b^{\prime} \rightarrow t^{\prime} W^{*}\right)$ could probe very tiny $V_{t b^{\prime}}$ values. This is because $b^{\prime} \rightarrow t^{\prime} W^{*}$ is phase space suppressed (as electroweak precision tests imply proximity of $\left|m_{t^{\prime}}-m_{b^{\prime}}\right| \lesssim M_{W}$ ), while $b^{\prime} \rightarrow t W$ is suppressed by $\left|V_{t b^{\prime}}\right|^{2}$. This effect is illustrated in the right plot of Fig. 2, where the threshold is when the $W^{*}$ turns on-shell. 

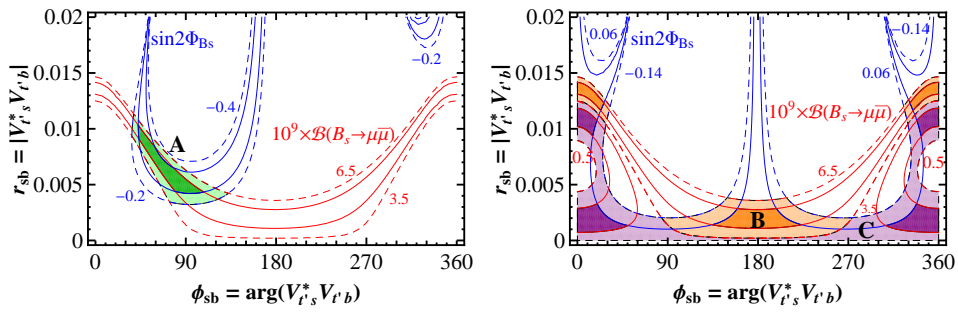

Figure 1: Overlap region for Cases A-C corresponding to Eqs. (2.3) and (2.4) as described in text.
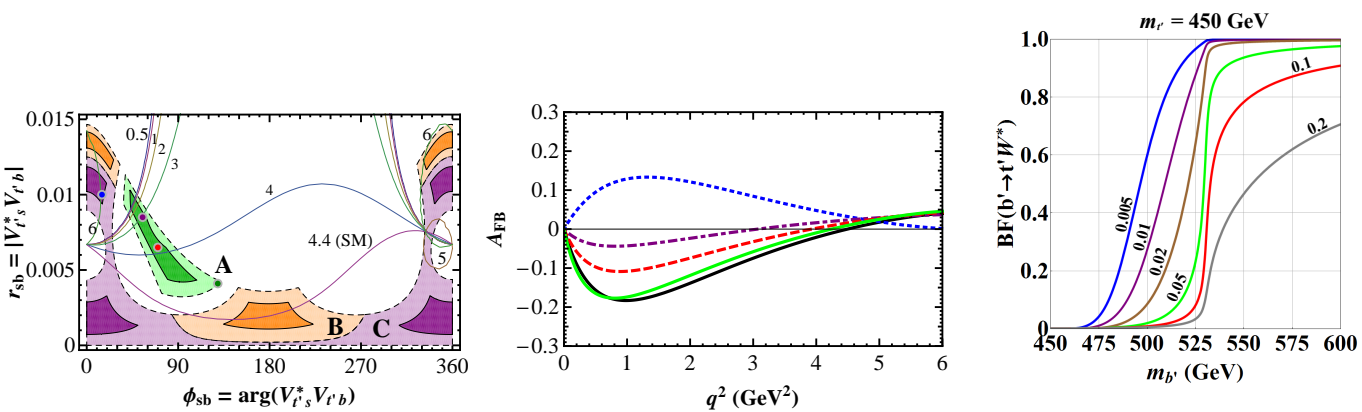

Figure 2: Left: $\left.s_{0} \equiv q^{2}\right|_{A_{\mathrm{FB}}=0}$ contours overlayed with Cases A-C; Center: $d A_{\mathrm{FB}} / d q^{2}$ vs $q^{2}$, where curves in descending order are for sample points (small ellipses) from high to low $r_{s b}$ in left plot, plus SM (black solid curve); Right: Probing very small $\left|V_{t b^{\prime}}\right|$ via $\mathscr{B}\left(b^{\prime} \rightarrow t^{\prime} W^{*}\right)$, i.e. transition between 4 th generation quarks.

\section{Conclusion: Towards $b \rightarrow s$ Quadrangle}

To illustrate the implications of our proposed program, we plot $V_{t^{\prime} s}^{*} V_{t^{\prime} b} \simeq 0.0065 e^{i 70^{\circ}}$ in Fig. 3 . This corresponds to both $\sin 2 \Phi_{B_{s}}$ and $\mathscr{B}\left(B_{s} \rightarrow \mu^{+} \mu^{-}\right)$on the large side allowed by current data. Note that $V_{u s}^{*} V_{u b}$ and $V_{c s}^{*} V_{c b}$ are measured by tree processes, and we assume CKM4 unitarity.

In conclusion, along with direct search of $t^{\prime}$ and $b^{\prime}$ quarks, the LHC could measure 4 th generation CKM parameters in the near future, which could bear the matter asymmetry of the Universe.

\section{References}

[1] Plenary talk by G. Tonelli, this proceedings.

[2] Plenary talk by A. De Roeck at Lepton Photon Symposium, August 2011, Mumbai, India.

[3] W.-S. Hou, Chin. J. Phys. 47, 134 (2009).

[4] W.-S. Hou, M. Nagashima and A. Soddu, Phys. Rev. D 76, 016004 (2007).

[5] A.J. Buras, Phys. Lett. B 566, 115 (2003).

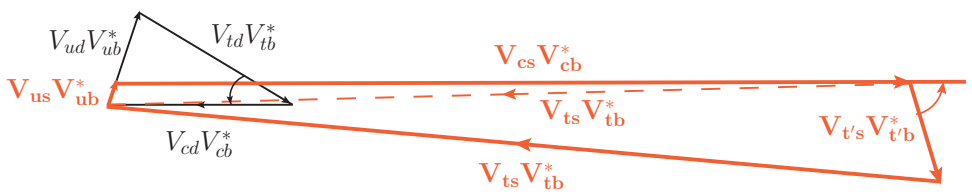

Figure 3: An illustrative $b \rightarrow s$ quadrangle of SM4, together with $b \rightarrow d$ and $b \rightarrow s$ triangles of SM3. 